
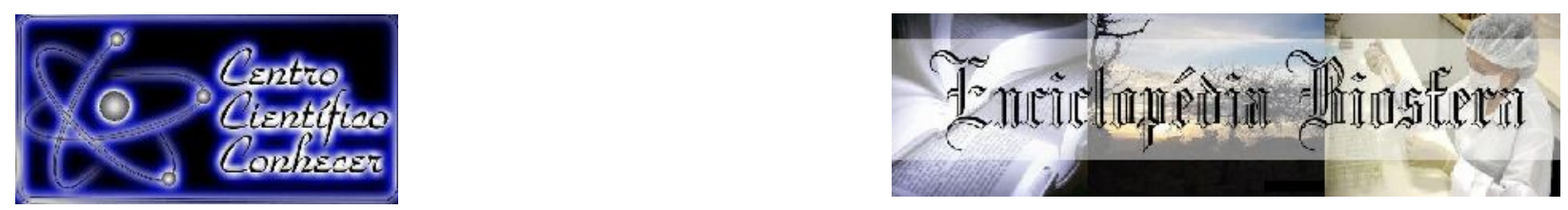

\title{
ARTROPODOFAUNA EDÁFICA DE UM SISTEMA AGROFLORESTAL (SAF) EM UM ASSENTAMENTO AGRÁRIO NA REGIÃO AMAZÔNICA
}

\author{
Ana Lucia Nunes Gutjahr ${ }^{1}$, Andreza Mesquita Martins ${ }^{2}$, Carlos Elias de Souza \\ Braga $^{1}$, Stephanie Santos Viana Santos ${ }^{3}$
}

1 Professor (a) Doutor(a), Universidade do Estado do Para, Departamento de Ciências Naturais (algutjahr@uepa.br), Belém, Para, Brasil.

2 Mestra em Ciências Ambientais, Universidade do Estado do Para, Belém-PA, Brasil.

3 Graduanda de Ciências Naturais: Biologia, Universidade do Estados do Para, Belém-PA, Brasil.

Recebido em: 15/08/2020 - Aprovado em: 15/09/2020 - Publicado em: 30/09/2020 DOI: 10.18677/EnciBio_2020C8

\begin{abstract}
RESUMO
O filo Arthropoda é considerado o maior agrupamento de seres vivos da Terra, estes animais possuem grande adaptabilidade e podem ser encontrados em diversos ambientes naturais e alterados. O objetivo deste trabalho foi estudar a composição da artropodofauna edáfica, durante os períodos sazonais da região amazônica em um Sistema agroflorestal. A amostragem foi realizada no Sistema Agroflorestal (SAF) da Associação de Trabalhadores Rurais Agroecológico Expedito Ribeiro (ATRAER), no período de marco e abril de 2018 (estação chuvosa) e de julho e agosto de 2018 (estação menos chuvosa). As coletas foram realizadas em uma área de $50 \times 25 \mathrm{~m}$ e o método de captura da artropodofauna edáfica foi a armadilha de queda (pitfall trap). Foram coletados 21.565 artrópodes edáficos, pertencentes a 16 ordens. A maior abundância foi observada na estação menos chuvosa, com 11.293 exemplares e 15 ordens coletados. Verificou-se a maior abundância e dominância para Collembola $(n=13.905 ; \mathrm{Fr}=64,48)$, Hymenoptera $(n=4.096 ; \mathrm{Fr}=18,99) \mathrm{e}$ Coleoptera $(n=1.383 ; \mathrm{Fr}=6,41)$. O SAF da ATRAER apresentou maior abundância de artrópodes edáficos na estação menos chuvosa e, os invertebrados dos grupos Diplopoda e Dermaptera são raros no ambiente estudado.
\end{abstract}

PALAVRAS-CHAVE: Agrofloresta. Artrópodes edáficos. Fauna de solo. Agroecologia.

\section{THE EDAPHIC ARTHROPODOFAUNA OF AN AGRO-FOREST SYSTEM (SAF) IN AN AGRARIAN SETTLEMENT IN THE AMAZON REGION}

\footnotetext{
ABSTRACT

The phylum Arthropoda is considered the largest grouping of living beings on Earth, these animals have great adaptability and can be found in several natural environments as well as the altered ones. The purpose of this work was to study the composition of the edaphic arthropodofauna, during the seasonal periods of the Amazon region in an agroforestry system. The sampling was carried out in the Agroforestry System (SAF) of the Association of Rural Workers Agroecological Expedito Ribeiro (ATRAER), between March and April 2018 (rainy season) and July and August 2018 (less rainy season). The gathering was carried out in an area of $50 \times 25 \mathrm{~m}$ and the method used to capture the edaphic arthropodofauna was the pitfall ENCICLOPÉDIA BIOSFERA, Centro Científico Conhecer - Jandaia-GO, v.17 n.33; p. $85 \quad 2020$
} 
trap. 21,565 edaphic arthropods were captured, from 16 different orders. The most abundance season was the least rainy season, which were collected 11,293 specimens from 15 orders. The greatest abundance and dominance was the Collembola ( $n=13,905 ; \mathrm{Fr}=64.48)$, Hymenoptera $(\mathrm{n}=4,096 ; \mathrm{Fr}=18.98)$ and Coleoptera $(n=1,383$; $\mathrm{Fr}=6.41)$. ATRAER Agroforestry systems showed the most abundance of edaphic arthropods in the less rainy season, and invertebrates from Diplopoda and Dermaptera groups are rare in the studied environment.

KEYWORDS: Agroforestry. Edaphic arthropods. Soil fauna. Agroecology.

\section{INTRODUÇÃO}

Os artrópodes são os animais que obtiveram o maior êxito evolutivo no planeta Terra, devido a sua grande capacidade adaptativa de sobrevivência em diferentes habitats terrestres (CAJAIBA; SILVA, 2014; ZACARIN et al., 2015). Este filo é composto por um grande número de espécies, sendo os mais familiares os insetos, as aranhas, escorpiões, centopeias, caranguejos, camarões, entre outros (BRAVO; CALOR, 2016). De acordo com Santos et al. (2018), os artrópodes ocupam uma grande diversidade de microhabitats e nichos ecológicos, isso faz com que eles possam desenvolver grande função ecológica nos ecossistemas.

Estes organismos exercem as mais variadas funções, como a decomposição de resíduos orgânicos e estruturação do solo, possuindo a capacidade de alterar as propriedades físicas, químicas e biológicas dos ambientes que habitam (ALMEIDA et al., 2017; NUNES-GUTJAHR et al., 2019). Por isso, pode-se afirmar que os artrópodes devido à alta diversidade de espécies e sua ligação física e biológica, são considerados bons bioindicadores da qualidade ambiental, sendo capazes de indicar o grau de alteração que ocorreu em um determinado ambiente (LEIVAS; FISCHER, 2007; AMARANTO et al., 2018).

Sabe-se que as alterações ambientes ocorrem de diversas formas, mas a que apresenta maior mudança na qualidade ambiental é o uso da terra ( $\mathrm{NUCCl}$ et al., 2019). Tal uso, afeta diretamente os seres vivos que necessitam da serrapilheira para desenvolver suas atividades biológicas e essas mudanças são causadas principalmente pelas monoculturas e o desflorestamento. Neste sentido, diversos estudos têm sido realizados com o intuito de identificar a composição faunística do solo, principalmente em sistemas de manejo agrícola, que promovam maior qualidade ambiental. Assim, os Sistemas Agroflorestais (SAF) ou agroflorestas, são sistemas de produção caracterizados por apresentar uma grande diversidade específica, devido à combinação de espécies florestais com cultivos agrícolas (LIMA et al., 2010; SANTOS et al., 2020).

Segundo Smiley e Kruschel (2008) e Barbosa et al. (2017), os SAF propiciam a deposição contínua de resíduos vegetais, o que facilita a manutenção da matéria orgânica do solo afetando diretamente seus atributos físicos, químicos e biológicos. Além disso, os SAF podem garantir a conservação da biodiversidade, o sequestro de carbono e a melhoria no controle de qualidade da água, proporcionando benefícios ambientais aos ecossistemas (NAIR, 2008).

Segundo Silva et al. (2014), estudos voltados para o levantamento e caracterização da fauna de solo são importantes, por permitir a compreensão das complexas relações existentes entre os diferentes organismos na natureza, assim como, a avaliação da qualidade do solo, além proporcionar o entendimento e a compreensão das dinâmicas dos sistemas de produção agrícola. Pelo exposto este trabalho teve por objetivo estudar a composição da artropodofauna edáfica, durante os períodos sazonais da região amazônica em um Sistema agroflorestal (SAF). 


\section{Área de estudo}

\section{MATERIAL E MÉTODOS}

O estudo foi realizado em uma área de Sistema Agroflorestal (SAF), localizada no município de Santa Bárbara do Pará, região metropolitana de Belém, Pará (Figura 1). Essa área corresponde a Associação de Trabalhadores Rurais Agroecológico Expedito Ribeiro (ATRAER) situada nas coordenadas 0121'60"S, 4826'40"W. Vale ressaltar que esta área é conhecida na região como Assentamento Expedito Ribeiro, situado na Rodovia PA 408.

FIGURA 1. Localização do município de Santa Bárbara do Pará, e ponto de coleta da artropodofauna edáfica no Sistema Agroflorestal (SAF) do Assentamento Expedito Ribeiro em 2018.

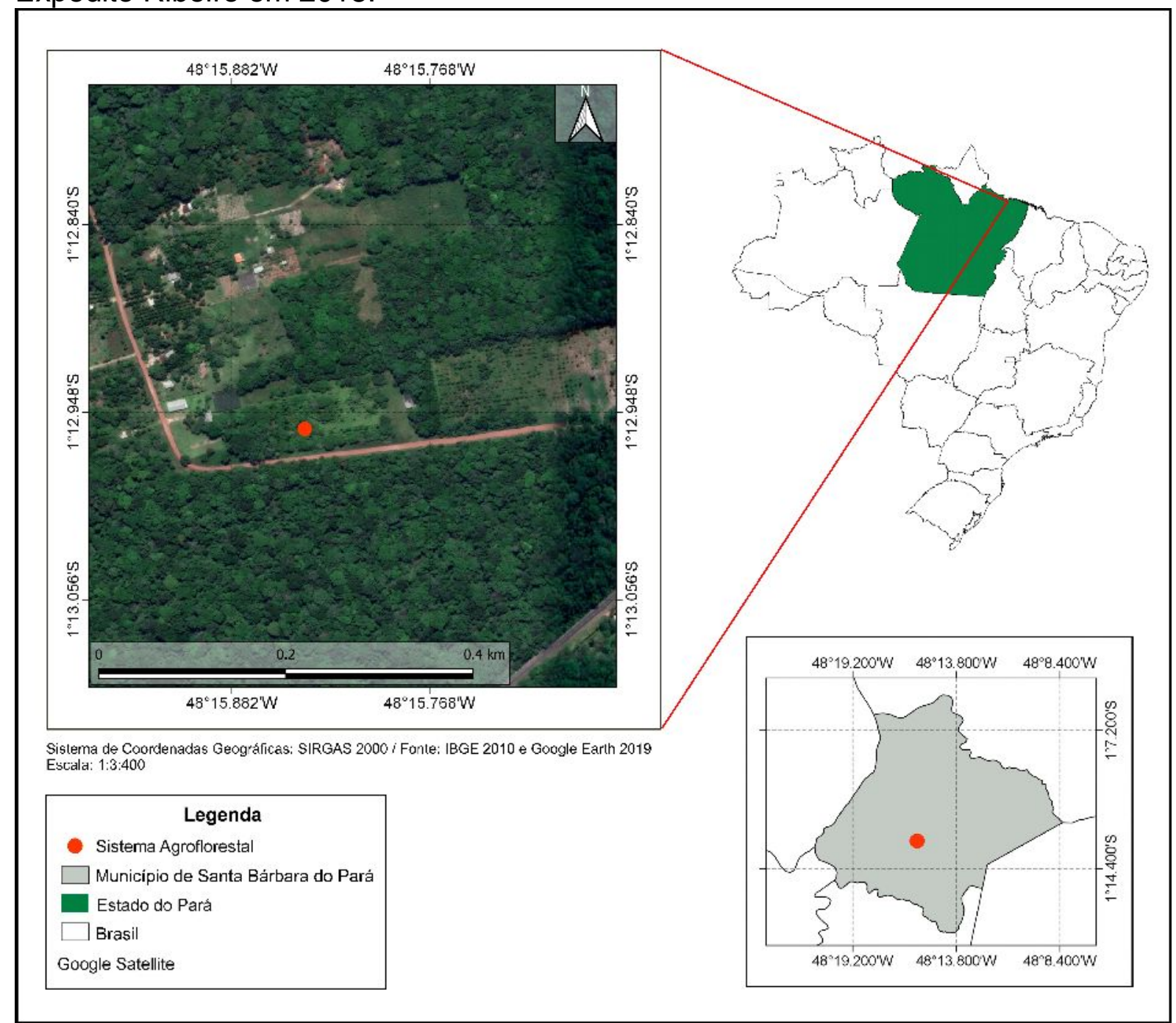

Fonte: Os autores (2020)

O SAF da ATRAER foi implantado em 2012, sendo, portanto, uma agrofloresta jovem. Nesse SAF estão cultivadas as espécies florestais, tais como: Taperebá (Spondias mombin L.), Ingá (Inga edulis Mart.), fruta pão (Artocarpus altilis (Parkinson) Fosberg), ipê (Tabebuia Alba (Cham.) Sandwith), freijó (Cordia goeldiana Huber), castanha-do-Pará (Bertholletia excelsa Bonpl.), bacuripari (Garcinia brasiliensis Mart.), rambutã (Nephelium lappaceum L.) e mogno brasileiro (Swietenia macrophylla King), sendo estes plantados com espaçamento de $10 \mathrm{~m}$ 
umas das outras. Dentre os frutos produzidos neste SAF, destacam-se a comercialização de cacau (Theobroma cacao L.), cupuaçu (Theobroma grandiflorum Schumann), acerola (Malpighia glabra L.), banana (Musa sp.) entre outros.

\section{Método de Coleta da Artropodofauna Edáfica}

A região amazônica tem dois períodos distintos, que evidenciam a sazonalidade regional: período chuvoso que ocorre de dezembro a maio e o período menos chuvoso, que se estende de junho a novembro. Por isso, a coleta da artropodofauna edáfica foi realizada em quatro excursões de campo: duas na estação chuvosa (marco e abril/2018), e duas na estação menos chuvosa (julho e agosto/ 2018).

As amostras foram obtidas em uma área de $50 \times 25 \mathrm{~m}$ onde foram estabelecidos dois transectos de $50 \mathrm{~m}$ que distavam entre si $25 \mathrm{~m}$. Em cada transecto foram definidos seis pontos de amostragens, que totalizaram 12 pontos amostrais na área de estudo, sendo que cada ponto estava distante entre si $10 \mathrm{~m}$.

A captura da artropodofauna edáfica foi obtida por meio de coletas com o uso de pitfall trap (armadilhas de queda). Essa armadilha é considerada a mais eficiente para a captura dos invertebrados que habitam o solo (BROWN; MATTHEWS, 2016; VALE JUNIOR et al., 2017). A armadilha de ptifall consiste em enterrar um copo plástico de $300 \mathrm{~mL}$ com a borda nivelada ao solo, e coberto por uma estrutura composta por palitos de madeira acoplados a um prato plástico que servia de telhado de proteção contra chuva. As armadilhas continham solução de álcool a $70 \%$ e ficaram ativas por 72 horas, sendo posteriormente transportadas para o laboratório em potes plásticos, devidamente tampados e identificados com as informações de coleta. Para este estudo, considerando as quatro excursões de campo, foram totalizadas 48 amostras.

\section{Identificação dos Espécimes}

A identificação e a quantificação da artropodofauna edáfica foi realizada com auxílio de um microscópio estereoscópico. Para a identificação em nível de ordem, foram utilizadas chaves dicotômicas de Triplehorn e Johnson (2015). Após a identificação, os espécimes coletados no SAF, foram quantificados etiquetados e acondicionados em tubos Eppendorf com solução de álcool $80 \%$ e se encontram depositados na Coleção Zoologica Dr. Joachim Adis da Universidade do Estado do Pará (CZJA/UEPA).

\section{Análise de Dados}

Os dados deste estudo foram analisados descritivamente e por meio do Teste t de Student (significância: $\alpha<0,05$; para verificar se havia diferença na abundância da artropodofauna, entre os períodos chuvoso e menos chuvoso), utilizando o pacote estatístico BioEstat 5.0. Além disto, foi realizado o índice de dominância, sendo consideradas as ordens dominantes aquelas que obtiveram valores de frequência relativa ( $\mathrm{Fr}$ ) maior ou igual $5 \%$, do total de espécimes inventariados (NUNES-GUTJAHR; BRAGA, 2015).

\section{RESULTADOS E DISCUSSÃO}

No Sistema agroflorestal da ATRAER foram coletados 21.565 espécimes de artrópodes edáficos, pertencentes a 16 ordens (Acari, Araneae, Blattodea, Chilopoda, Coleoptera, Collembola, Isopoda, Dermaptera, Diplopoda, Diptera, Hemiptera, Hymenoptera, Opiliones, Orthoptera, Psocoptera e Thysanoptera) 
(Tabela1). Entre os artrópodes edáficos coletados, a ordem que apresentou maior abundância foi Collembola $(n=13.905)$, seguida de Hymenoptera $(n=4.096)$ e Coleoptera $(n=1.383)$, enquanto as ordens com menor abundância foram Chilopoda e Dermaptera, ambos com apenas um espécime e Diplopoda $(n=9)$ (Tabela 1).

TABELA 1 - Ordens, Frequência relativa $(F r)$ e número de espécimes da artopodofauna edáfica coletados por período sazonal em um Sistema Agrofolorestal (SAF), Santa Bárbara do Pará, em 2018.

\begin{tabular}{|c|c|c|c|c|c|c|}
\hline \multirow[b]{2}{*}{ ORDEM } & \multicolumn{4}{|c|}{ Período } & \multirow{2}{*}{$\begin{array}{c}\text { Total de } \\
\text { exemplares }\end{array}$} & \multirow[b]{2}{*}{$F(\%)$} \\
\hline & Chuvoso & $\mathbf{F}(\%)$ & $\begin{array}{c}\text { Menos } \\
\text { chuvoso }\end{array}$ & F (\%) & & \\
\hline Acari & 299 & 2.91 & 591 & 5.23 & 890 & 4.13 \\
\hline Araneae & 197 & 1.92 & 41 & 0.36 & 238 & 1.10 \\
\hline Blattodea & 32 & 0.31 & 9 & 0.08 & 41 & 0.19 \\
\hline Chilopoda & 0 & 0.00 & 1 & 0.01 & 1 & 0.00 \\
\hline Coleoptera & 825 & 8.03 & 558 & 4.94 & 1383 & 6.41 \\
\hline Collembola & 6217 & 60.52 & 7688 & 68.08 & 13905 & 64.48 \\
\hline Isopoda & 84 & 0.82 & 25 & 0.22 & 109 & 0.51 \\
\hline Dermaptera & 0 & 0.00 & 1 & 0.01 & 1 & 0.00 \\
\hline Diplopoda & 1 & 0.01 & 8 & 0.07 & 9 & 0.04 \\
\hline Diptera & 151 & 1.47 & 180 & 1.59 & 331 & 1.53 \\
\hline Hemiptera & 208 & 2.02 & 168 & 1.49 & 376 & 1.74 \\
\hline Hymenoptera & 2150 & 20.93 & 1946 & 17.23 & 4096 & 18.99 \\
\hline Opiliones & 31 & 0.30 & 0 & 0.00 & 31 & 0.14 \\
\hline Orthoptera & 56 & 0.55 & 44 & 0.39 & 100 & 0.46 \\
\hline Psocoptera & 16 & 0.16 & 17 & 0.15 & 33 & 0.15 \\
\hline Thysanoptera & 5 & 0.05 & 16 & 0.14 & 21 & 0.10 \\
\hline TOTAL & 10272 & - & 11293 & - & 21565 & - \\
\hline
\end{tabular}

Em relação a maior abundância das ordens Collembola e Hymenoptera (principalmente formigas), observadas neste estudo, as quais correspondem aos colêmbolos ou pulgas-da-terra e as formigas, pode-se admitir que dentro da fauna de solo esses são os componentes mais efetivos e atuantes, já que as duas ordens reúnem os principais grupos da artropodofauna. Tal resultado, é corroborado pelo trabalho realizado por Nunes-Gutjahr et al. (2019), no qual foi também observada maior abundância das ordens Collembola, Hymenoptera e Coleoptera em uma plantação de dendê, no município de Santa Bárbara do Pará, e por Pompeo et al. (2016), que observaram a prevalência de Collembola, Coleoptera e Hymenoptera na fauna edáfica em ambientes de campos nativos no município de Lages, em Santa Catarina. 
Quanto à ordem Coleoptera, que também foi abundante neste estudo, podese afirmar que, os representantes desta ordem possuem potencial para atuarem como um dos principais grupos de predadores no solo em diversos cultivos agrícolas (LEMOS, 2011), o que favorece o controle de populações de diversos outros invertebrados edáficos, mantendo, por isso, o equilíbrio das comunidades edáficas.

Em relação aos dois períodos de pluviosidade amazônica, a maior abundância de indivíduos foi observada durante a estação menos chuvosa, com 11.293 exemplares coletados (pertencentes a 15 ordens), em comparação com a estação chuvosa (Tabela 1; Figura 2). Quanto a esse resultado, pode-se afirmar que o maior número de exemplares coletados na estação menos chuvosa, foi confirmado estatisticamente, visto que, observou-se a existência de diferença significativa entre ambos os períodos amostrados (teste $t, p<0,05$ ). Tal resultado pode indicar que 0 índice pluviométrico parece ser um fator limitante para artropodofauna no sistema agroflorestal. Quanto a isso, Corrêa et al. (2011), em Rondônia, também verificaram maior abundância de invertebrados edáficos no período seco, em um Sistema Agroflorestal, e justificaram que tal relação ocorreu provavelmente devido a maior liberação de serrapilheira pela vegetação durante a estação seca, em relação a estação chuvosa.

FIGURA 2. Número de espécimes coletados por período de pluviosidade amazônica, da artropodofauna edáfica no Sistema Agroflorestal (SAF), em 2018.

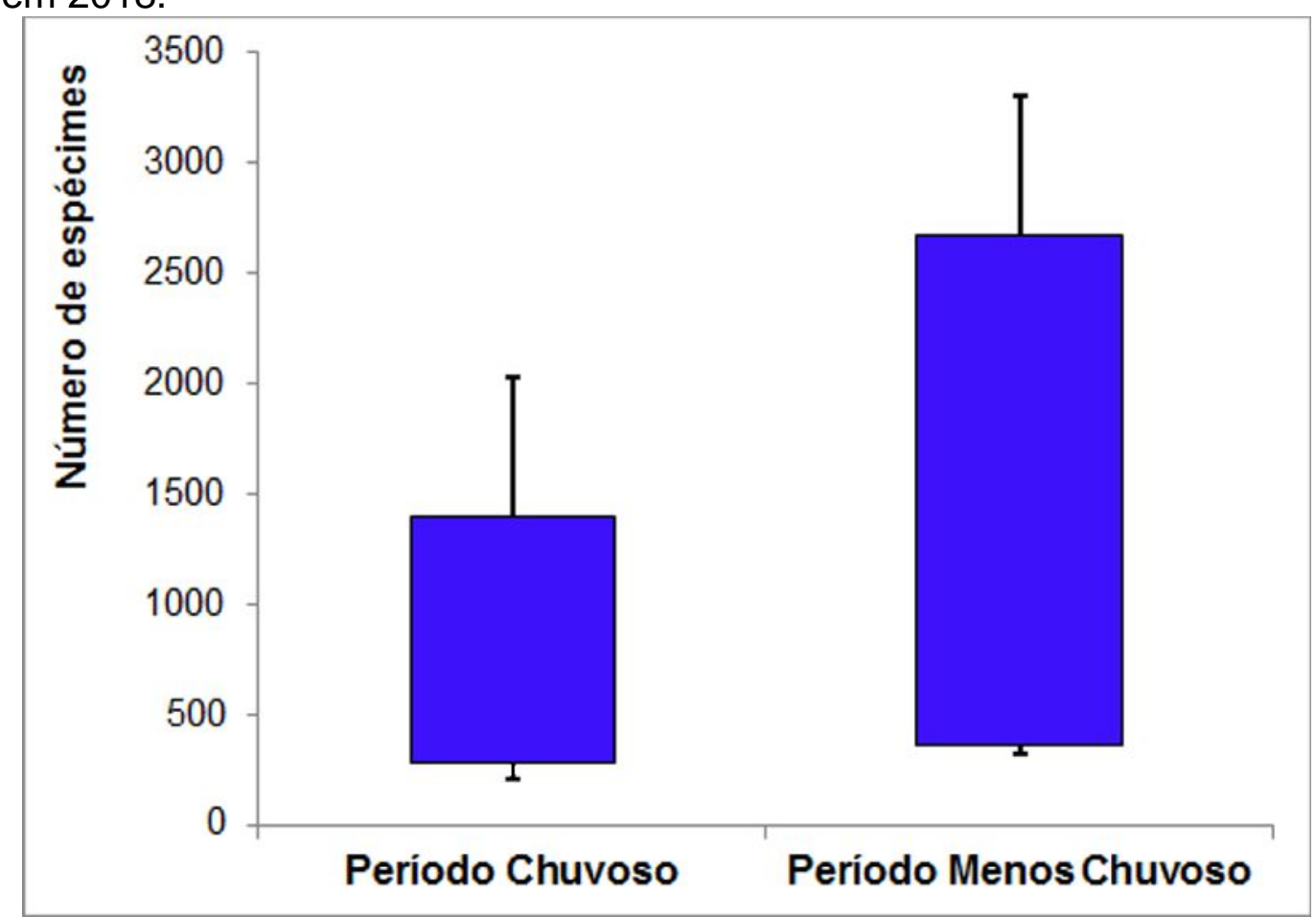

Fonte: Os autores (2020)

Além disto, observou-se que dentre as ordens, apenas Chilopoda e Dermaptera não apresentaram ocorrência no período chuvoso, além de Opiliones no período menos chuvoso, visto que as demais ordens ocorreram em ambos os períodos (Tabela 1). Isso pode ser um indicativo de que os artrópodes da Classe Chilopoda e os insetos da ordem Dermaptera possam ser sensíveis ao maior nível de umidade do solo característico da estação chuvosa, o que de modo contrário ocorreu para o aracnídeo da ordem Opiliones. Entretanto, o número de Chilopoda e 
Dermaptera amostrados foi pequeno $(n=1)$, representando apenas unicatas em todo período de amostras, o que pode indicar raridade desses animais no ambiente do SAF, visto que, este por ser jovem possui uma cobertura de dossel aberto, devido ao porte da vegetação e também, pela pouca quantidade de serapilheira no solo, o que torna o solo desfavorável para esses invertebrados predadores. Tal constatação pode ser corroborada por um estudo de Silva et al. (2018) no qual verificaram que o estoque de serapilheira em florestas na fase de restauração com menos de cinco anos eram significativamente menores em comparação às florestas com mais de 40 anos, demonstrando que nesses ambientes havia uma menor quantidade de nutrientes e consequentemente menor diversidade.

Considerando a frequência relativa (Dominância $=\mathrm{Fr}>5 \%$ ) dos grupos taxonômicos da artropodofauna entomológica estudada, os resultados evidenciam a dominância das ordens: Collembola $(\mathrm{Fr}=64,48)$, Hymenoptera $(\mathrm{Fr}=18,99)$ e Coleoptera $(\mathrm{Fr}=6,41)$. Durante o período chuvoso as mesmas ordens descritas anteriormente foram dominantes Collembola $(\mathrm{Fr}=60,52)$, Hymenoptera $(\mathrm{Fr}=20,93)$ e Coleoptera $(\mathrm{Fr}=8,03)$. Já no período menos chuvoso observou-se a dominância das ordens Collembola ( $\mathrm{Fr}=68,08)$, Hymenoptera $(\mathrm{Fr}=17,23)$ e Acari $(\mathrm{Fr}=5,23)$ (Tabela 1). Tal resultado, possivelmente se deve ao fato destes invertebrados serem exclusivamente edáficos, sendo, portanto, os grupos mais representativos dentro da artropodofauna no solo.

A grande dominância da ordem Collembola é devido principalmente por seus representantes estarem associados as dinâmicas do solo e serem um dos grupos de artrópodes mais abundantes no solo. Segundo Klein et al. (2016), estes invertebrados desempenham importantes funções no sistema edáfico, pois podem realizar a decomposição da matéria orgânica, a ciclagem de nutrientes, a regulação de populações microbianas, aumentam a porosidade do solo, contribuem para a troca de gases e a retenção de água, além do mais, essa ordem de artrópodes, é uma indicadora da qualidade ambiental.

Quanto à dominância da ordem Hymenoptera, observada neste estudo, podese admitir, que tal resultado já era esperado, pois as formigas como representantes deste grupo, possuem ampla distribuição espacial e geográfica e apresentam dominância numérica, nos ambientes onde ocorrem (KORASAKI et al., 2013). Além disso, as formigas facilmente se adaptam às diferentes condições locais, podendo haver predomínio de uma ou mais espécies (BRUYN, 1999).

A partir dos resultados obtidos neste estudo, foi possível verificar que a área do SAF estudado possui uma significativa diversidade de sua artropodofauna edáfica, apesar de ser uma área que se encontra em processo de sucessão ecológica, que ainda está recuperando sua fauna e o equilíbrio de suas dinâmicas e relações tróficas nos diferentes nichos existentes.

\section{CONCLUSÃO}

O Sistema Agroflorestal (SAF) da Associação de Trabalhadores Rurais Agroecológico Expedito Ribeiro (ATRAER), quanto à sazonalidade, foi evidente que a maior abundância de artrópodes edáficos ocorreu no período menos chuvoso, em relação ao período chuvoso amazônico.

Os grupos com maior abundância e dominância durante o período de amostragem, pertencem às ordens Collembola, Hymenoptera e Coleoptera, indicando que estes são os principais artrópodes edáficos no SAF da ATRAER, em contrapartida aos grupos de Diplopoda e Dermaptera que são considerados raros nesse ambiente. 


\section{AGRADECIMENTOS}

Os autores agradecem a Coordenação de Aperfeiçoamento de Pessoal de Nível Superior (CAPES) pela bolsa de mestrado; ao Conselho Nacional de Desenvolvimento Cientifico e Tecnológico (CNPq) pela bolsa de Iniciação Cientifica e a Associação de Trabalhadores Rurais Agroecológico Expedito Ribeiro (ATRAER).

\section{REFERÊNCIAS}

ALMEIDA, A. B.; ZACARIN, G. G.; SMITH, W. S. Inventário da biodiversidade de lacraias (Arthropoda, Chilopoda) em parques ecológicos do município de Sorocaba, São Paulo, Brasil. Journal of the Health Science Institute, v. 35, n. 2, p. 75-79, 2017. em:

https://www.unip.br/presencial/comunicacao/publicacoes/ics/edicoes/2017/02_abrjun/V35_n2_2017_p75a79.pdf>.

AMARANTO, I.; SALIMON, C. I.; ZEPPELINI, D. Relação entre massa corporal e taxa respiratória em Arthropoda de Floresta Atlântica (Paraíba, Brasil). Pesquisa e Ensino em Ciências Exatas e da Natureza, v. 2, n. 2, p. 119-129, 2018. Disponível em: < http://www.revistas.ufcg.edu.br/cfp/index.php/RPECEN/article/view/1066/438>.

BARBOSA, J. S.; SILVA, K. C. R.; CARDUCCI, C. E.; SANTOS, K. L.; KOHN, L. S.; FUCKS, J. S. Atributos Físico-hídricos de um Cambissolo Húmico Sob Sistema Agroflorestal no Planalto Catarinense. Floresta e Ambiente, v. 24, p. 1-9, 2017. Disponível em: < http://dx.doi.org/10.1590/2179-8087.025116>. DOI: 10.1590/21798087.025116.

BRAVO, F.; CALOR, A. R. Conhecendo os artrópodes do Semiárido. 1.ed. São Paulo: Métis Produção Editorial, p.192, 2016.

BROWN, G. R.; MATTHEWS, I. M. A review of extensive variation in the design of pitfalltraps and a proposal for a standard pitfall trap design formonitoring groundactive arthropod biodiversity. Ecology and Evolution, v. 6, n. 12, p. 3953-3964, 2016. Disponível em: < https://doi.org/10.1002/ece3.2176>. DOI: 10.1002/ece3.2176

BRUYN, L. Ants as bioindicators of soil function in rural environments. Agriculture, Ecosystems \& Environment, Zurich, v.74, p. 425-441, junho, 1999.

CAJAIBA, R. L.; SILVA, W. B. Percepção dos alunos do Ensino Fundamental sobre os insetos antes e após aulas práticas: um estudo de caso no município de UruaráPará, Brasil. Enciclopédia Biosfera, v.10, n.19; p. 2510-2521, 2014. Disponível em: <http://conhecer.org.br/enciclop/2014b/CIENCIAS\%20HUMANAS/percepca0\%20dos \%20alunos.pdf>. Acesso em: 13 ago. 2020.

CORRÊA, F. L. O.; CAPRONI, A. L.; GRANHA, J. R.; MULLER, M. W. Fauna edáfica em sistema agroflorestal multiestratificado em Rondônia, Brasil. Agrotrópica, v. 23, n. 2,3, p. 125-134, 2011. Disponível em:< http://snida.agricultura.gov.br/binagri/bases/agb/Agb_Docs_Fonte/BR2012003412.pd $\mathrm{f}>$. 
KLEIN, I; CERICATO, A; PREUSS, J. F. Entomofauna associada à cultura de milho transgênico (Bt) e convencional no município de Iraceminha, Santa Catarina, brasil. Unoesc \& Ciência - ACET, Joaçaba, v. 7, p. 167-173, 2016.

KORASAKI, V.; MORAIS, J. W.; BRAGA, R. F. Macrofauna. In: MOREIRA, F. M. S.; CARES, J. E.; ZANETTI, R.; STÜRMER, S. L. (Eds.). O ecossistema solo: componentes, relações ecológicas e efeitos na produção vegetal. Lavras: Editora da UFLA, p. 79-128, 2013.

LEIVAS, F. W. T.; FISCHER, M. L., Avaliação da composição de invertebrados terrestres em uma área rural localizada no município de Campina Grande do Sul. Paraná, Brasil, Revista Biotemas, p. 65-73, mar/ 2008.

LEMOS, W. P.; LIMA, M. M.; SANTO, L. N. E.; BISPO, C. J. C. Artropodofauna de solo com potencial de uso em programas de controle biológico de pragas em cultivos alternativos de palma de óleo (Elaeis guineensis). Embrapa Amazônia Oriental. Parte de Livro. 2011. Disponível em: https://www.embrapa.br/busca-de-publicacoes//publicacao/910555/artropodofauna-de-solo-com-potencial-de-uso-em-programasde-controle-biologico-de-pragas-em-cultivos-alternativos-de-palma-de-oleo-elaeisguineensis. Acessado em: 27 de março de 2020.

LIMA, S. S.; LEITE, L. F. C.; AQUINO, A. M.; OLIVEIRA, F. C.; CASTRO, A. A. J. F. Estoques da serrapilheira acumulada e teores de nutrientes em Argissolo sob manejo agroflorestal no norte do Piauí. Revista Árvore, v. 34, p. 75-84, 2010.

NAIR, P. K. R. Agroecosystem management in the 21st century: it is time for a paradigm shift. Journal of Tropical Agriculture, v. 46, p. 1-12, 2008.

NUNES-GUTJAHR, A. L.; BRAGA, C. E. Analise faunística de gafanhotos Acridoidea da Volta Grande do Rio Xingu, área de influência direta da Hidrelétrica Belo Monte, Para, Brasil. Ciência Rural, v. 45, n. 7, p.1220-1227, 2015. Disponível em: <http://dx.doi.org/10.1590/0103-8478cr20120442>

NUNES-GUTJAHR, A. L.; MARTINS, A. M.; BRAGA, C. E. BORGES, H. S.; SANTOS, S. S. V. A Entomofauna Edáfica em Cultura de Dendê no Município de Santa Bárbara, Pará, Brasil. Enciclopédia Biosfera, v.16 n. 29; p.17, 2019. Disponível em: DOI: 10.18677/EnciBio_2019A2

NUCCI, J. C.; VALASKI, S.; ESTÊVEZ, L. F.; TONETTI, E. L. Uso da terra e qualidade ambiental urbana: uma proposta de legenda para mapeamento. Geographia, v. 21, n. 46, p. 73-90, 2019. Disponível em: DOI: https://doi.org/10.22409/GEOgraphia2019.v21i46.a14390.

POMPEO, P. N.; SANTOS, M. A. B.; BIASI, J. P.; SIQUEIRA, S. F.; ROSA, M. G.; BARETTA, C. R. D. M.; BARETTA5, D. Fauna e sua relação com atributos edáficos em Lages, Santa Catarina - Brasil. Revista Scientia Agraria, v. 17, n. 1, p. 42-51, 2016. Disponível em:<http://dx.doi.org/10.5380/rsa.v17i1.46535>. DOI: 10.5380/rsa.v17i1.46535. 
SANTOS, M.; ALMEIDA, R.; ANTUNES, S. C. Artrópodes. Revista de Ciência Elementar, $\quad$ v. 6 , n. 2, p. 1-5, 2018. Disponível em: <https://doi.org/10.24927/rce2018.042>. DOI: 10.24927/rce2018.042.

SANTOS, W. M.; FARIA, L. R.; ROCHA, A. F. M.; VALE, L. S. R.; KRAN, C. S. Sistema Agroflorestal na Agricultura Familiar. Revista UFG, v. 20, p. 1-19, 2020. Disponível em: < DOI: https://doi.org/10.5216/revufg.v20.63772>. DOI: 10.5216/REVUFG.V20.63557.

SILVA, A. B.; GADELHA, B. Q.; RIBEIRO, A. C.; FERRAZ, A. C. P.; AGUIAR, V. M. Entomofauna capturada em armadilha para dípteros na Reserva Biológica do Tinguá, Nova Iguaçu, Rio de Janeiro. Bioikos, Campinas, v. 28, n. 1, p. 11-23, 2 janeiro/junho, 2014.

SILVA, K. A.; MARTINS, S. V.; LOPES, A. T. Estoque de serapilheira em uma floresta em processo de restauração após mineração de bauxita. Rodriguésia, v. 69, n. 2, p. , 2018. Disponível em:< https://doi.org/10.1590/2175-7860201869240>. DOI: $10.1590 / 2175-7860201869240$.

SMILEY, G. L.; KROSCHEL, J. Temporal change in carbon stocks of cocoa-gliricidia agroforests in Central Sulawesi, Indonesia. Agroforestry System, v. 73, p. 219-231, 2008.

TRIPLEHORN, C. A.; JOHNSON, N. F. Estudo dos Insetos. Tradução da 7음 Edição de Borro and Delong's introduction to the study of insects. $2 a$ ed., Cengage Learning. São Paulo, p. 766, 2015.

VALE JUNIOR, J. F.; LIMA, A. C. S.; CIDADE, M. P. N.; BANDEIRA, H. F. S.; CRUZ, D. L. S. Composição da assembleia de formigas em área de savana no norte da Amazônia. Revista Agroambiente On-line, v. 11, n. 2, p. 153-162, 2017. Disponível em: < https://revista.ufrr.br/agroambiente/article/view/3813/2269>.

https://dx.doi.org/10.18227/1982-8470ragro.v11i2.3813

ZACARIN, G. G.; RIBEIRO, S. M.; BARROS, R.; ALMEIDA, A. B. Invertebrados terrestres. In SMITH, W. S.; RIBEIRO, C. A. (orgs.) Parque Natural Municipal Corredores de Biodiversidade: pesquisas e perspectivas futuras. Secretaria do Meio Ambiente. p. 135-144, 2015 